\title{
WEAKLY COMPACT OPERATORS AND THE STRONG* TOPOLOGY FOR A BANACH SPACE
}

\author{
ANTONIO M. PERALTA, IGNACIO VILLANUEVA, J. D. MAITLAND WRIGHT, \\ AND KARI YLINEN
}

\begin{abstract}
The strong* topology $s^{*}(X)$ of a Banach space $X$ is defined as the locally convex topology generated by the seminorms $x \mapsto\|S x\|$ for bounded linear maps $S$ from $X$ into Hilbert spaces. The w-right topology for $X, \rho(X)$, is a stronger locally convex topology, which may be analogously characterised by taking reflexive Banach spaces in place of Hilbert spaces. For any Banach space $Y$, a linear map $T: X \rightarrow Y$ is known to be weakly compact precisely when $T$ is continuous from the w-right topology to the norm topology of $Y$. The main results deal with conditions for, and consequences of, the coincidence of these two topologies on norm bounded sets. A large class of Banach spaces, including all $C^{*}$-algebras, and more generally, all $J B^{*}$-triples, exhibit this behaviour.
\end{abstract}

\section{IntroduCtion, BACKGROUND, AND NOTATION}

A celebrated theorem of Davis, Figiel, Johnson, and Pełczyński [7] says that for Banach spaces $X$ and $Y$, a linear map $T: X \rightarrow Y$ is weakly compact if and only if there is a reflexive Banach space $E$ with bounded linear maps $R: X \rightarrow E$ and $S: E \rightarrow Y$ such that $T=S R$. In other words, the operator ideal, $\mathcal{W}$, of all weakly compact operators between Banach spaces coincides with the ideal of all operators which are factorisable through reflexive spaces. Operators factoring through Hilbert spaces constitute another example of operator ideal (denoted by $\Gamma_{2}$ ).

The operator ideals $\mathcal{W}$ and $\Gamma_{2}$ have been shown very useful to define several topologies on a Banach space. We denote for a moment, following [25], by $\mathcal{P}(\mathcal{W})(X)$ the locally convex topology on $X$ generated by the seminorms of the form $x \mapsto\|S x\|$ where $S$ is a bounded linear map from $X$ to a reflexive Banach space, and by $\mathcal{P}\left(\Gamma_{2}\right)(X)$ the analogous topology where now $S$ is always a bounded linear map into a Hilbert space. Then a linear map $T: X \rightarrow Y$ factors through a reflexive space (resp. a Hilbert space) if and only if it is continuous from $\mathcal{P}(\mathcal{W})(X)$ (resp. from $\mathcal{P}\left(\Gamma_{2}\right)(X)$ ) to the norm topology (see [25] or $\S 2$ below). We consider these topologies (with

First author partially supported by I+D MCYT project no. MTM 2008-02186, and Junta de Andalucía grants FQM 0199, 1215 and second author partially supported by I+D MCYT project no. MTM 2005-00082. 
different names) below, and results concerning their interconnections will be discussed.

If $X$ is taken to be a commutative unital $C^{*}$-algebra, seeds for some basic answers and precedents dealing with weak compactness vs. Hilbert spaces, are more than half a century old. In [3] Bartle, Dunford, and Schwartz showed that a weakly compact operator $T$ on $X$ comes with a measure, a positive linear form, that controls the behaviour of $T$ in a useful way. And once there is a measure, an $L^{2}$-space is not far behind. On the other hand, given an abstract Hilbert space $H$ and a bounded linear map $T: X \rightarrow H$, we have a bounded bilinear form $(x, y) \mapsto(T x \mid T y *)$, whose nature is largely explained by the so-called little Grothendieck inequality dating back to [10], again yielding measures and $L^{2}$-norms.

With the growth of noncommutative - also vector valued - measure theory, vast new areas were cultivated, but here we confine our attention to just one line of development. In [13] Jarchow showed, generalising an approach due to Jarchow and Pełczyński, already seen in the commutative case in [12], that for a $C^{*}$-algebra $A$ and a Banach space $Y$, a linear map $T: A \rightarrow Y$ is weakly compact if and only if there exist a Hilbert space $H$, a bounded linear map $Q: A \rightarrow H$, and for each $\epsilon>0$ a number $N(\epsilon)>0$ such that $\|T x\| \leq N(\epsilon)\|Q x\|+\epsilon\|x\|$ for all $x \in A$. The proof hinges on the noncommutative extension, due to Akemann [1], of the Bartle-DunfordSchwartz control measure result, thus producing the required Hilbert space as an analogue of an $L^{2}$-space.

If one wants to extend Jarchow's theorem to (at least some) more general Banach spaces than just $C^{*}$-algebras, the first step is to replace the noncommutative control measure result of Akemann by a pure Banach space condition. In Akemann's work, a key role is played by seminorms of the form $x \mapsto \phi\left(x^{*} x+x x^{*}\right)^{\frac{1}{2}}$ where $\phi$ ranges over the positive linear forms on the $\mathrm{C}^{*}$ algebra. While this looks like an specific $C^{*}$-algebra situation, from the point of view of locally convex topologies it is not. Indeed, as a consequence of the noncommutative generalisation of the little Grothendieck inequality, see [11], [19], the locally convex topology on a $C^{*}$-algebra $A$ generated by the seminorms $x \mapsto \phi\left(x^{*} x+x x^{*}\right)^{\frac{1}{2}}$ coincides with the one generated by all seminorms of the form $x \mapsto\|S x\|$ where it is required that $S$ is a bounded linear map from $A$ into a Hilbert space. (There is an analogous theory for $J B^{*}$-triples; we give a brief discussion in $\S 3$ below.)

If $A$ is a $C^{*}$-algebra, the locally convex topology on $A$ generated by the seminorms of the form $x \mapsto \phi\left(x^{*} x+x x^{*}\right)^{\frac{1}{2}}$ where $\phi$ ranges over the positive linear forms on $A$, is the same as the strong* operator topology in the universal representation of $A$ (and actually agrees with the $\sigma$-strong* operator topology in this particular case). It is induced by what Sakai [23, p. $20]$ denotes by $s^{*}\left(A^{* *}, A^{*}\right)$ and calls the strong* topology of the $W^{*}$-algebra $A^{* *}$. In keeping with this tradition, for any Banach space $X$, we call the 
topology denoted by $\mathcal{P}\left(\Gamma_{2}\right)(X)$ above, the strong* topology and denote it by $s^{*}\left(X, X^{*}\right)$ or simply by $s^{*}(X)$.

We now discuss the topology denoted by $\mathcal{P}(\mathcal{W})(X)$ above. It is known that identifying $X$ with its canonical embedding in $X^{* *}$, the $\mathcal{P}(\mathcal{W})(X)$ topology agrees with the relative topology induced on $X$ by the Mackey topology, $m\left(X^{* *}, X^{*}\right)$, of $X^{* *}$. There are various ways of seeing this. One way is to reduce it, by a method already used in an early version of the present note and appearing in print in [16, Proposition 2.2], to [17, Proposition 2, §4]. Another proof is given by Wright [27]. Jarchow, [13, p. 343], states this as "clear" in the $C^{*}$-algebra context he studied. In [18] the topology on $X$ induced by $m\left(X^{* *}, X^{*}\right)$ was called the right topology; it was there shown that the weakly compact operators from $X$ to any Banach space $Y$ are precisely those which are continuous from this topology to the norm topology of $Y$. After the publication of [18], W. Ruess has kindly pointed out to us that this result, in fact the equivalence of (i), (ii) and (iii) in [18, Corollary 5], follows as a consequence of [21, Proposition 2.6 and Theorem 3.2], proved there in a more general setting.

Here we use the notation $\rho(X)$ or $\rho\left(X, X^{*}\right)$ for $\mathcal{P}(\mathcal{W})(X)$ (see $\S 2$ for an elaboration), but make a slight specification in the terminology: The relative $m\left(X^{* *}, X^{*}\right)$-topology will be called the weak compactness right or w-right topology on $X$.

We shall have occasion to consider the strong* and w-right topologies also in a restricted sense. Throughout the paper, $F$ denotes a closed separating subspace of the dual $X^{*}$ of $X$. We denote by $\rho(X, F)$ the locally convex topology of $X$ generated by the seminorms $x \mapsto\|S x\|$ where $S$ is a $\sigma(X, F)$ to- $\sigma\left(E, E^{*}\right)$-continuous (and hence, by the uniform boundedness principle, bounded) linear map from $X$ to a reflexive Banach space $E$. Analogously, we get the topology $s^{*}(X, F)$ if in this description the words "a reflexive Banach space" are replaced by "a Hilbert space". Thus $\rho\left(X, X^{*}\right)$ is simply the w-right topology of $X$ and $s^{*}\left(X, X^{*}\right)$ is its strong* topology. As noted before, we often denote $s^{*}\left(X, X^{*}\right)$ just by $s^{*}(X)$ and $\rho\left(X, X^{*}\right)$ by $\rho(X)$. (Note that our notation $s^{*}(X, F)$ is consistent with the notation used in [17] in a special case.)

One of the key results of [1] states that for a von Neumann algebra $\mathcal{M}$ the strong* operator topology agrees with the Mackey topology $m\left(\mathcal{M}, \mathcal{M}_{*}\right)$ on norm bounded subsets of $\mathcal{M}$. If a $C^{*}$-algebra $A$ is embedded in its second dual, this shows that the topologies $s^{*}(A)$ and $\rho(A)$ coincide on bounded sets. A fundamental question to be studied in this paper is to what extent this state of affairs carries over to more general Banach spaces.

The philosophy is that when a general Banach space $X$ has the property that $s^{*}(X)$ and $\rho(X)$ coincide on the norm closed unit ball of $X$, then we may expect that $X$ will have some of the good (Banach space) behaviour of operator algebras. We give a large class of Banach spaces for which these 
topologies coincide on their unit ball; this class includes $C^{*}$-algebras, $J B^{*}$ triples, spaces of bounded linear operators between Hilbert spaces and some other classical spaces.

\section{Notation}

Throughout the rest of the paper, unless specified, $X$ will stand for a general Banach space while $F$ will denote an arbitray closed separating subspace of the dual $X^{*}$ of $X$. The closed unit ball of $X$ will be denoted by $B_{X}$ with a similar usage for other Banach spaces. Unless stated otherwise or clear from the context, the scalar field may be either $\mathbb{R}$ of $\mathbb{C}$; we may use for them the common name $\mathbb{K}$.

\section{BASIC PROPERTIES OF THE STRONG* AND W-RIGHT TOPOLOGIES}

We give for the convenience of the reader a quick proof for the following observation, though it can also be deduced from the much more general ideas in the theory of operator ideals presented in [25].

Proposition 2.1. Let $Y$ be a Banach space and $T: X \rightarrow Y$ a linear map. The following conditions are equivalent:

(i) The map $T$ is $s^{*}(X, F)$-to-norm continuous.

(ii) There is a Hilbert space $H$ with a $\sigma(X, F)$-to- $\sigma\left(H, H^{*}\right)$-continuous linear map $R: X \rightarrow H$ and a bounded linear map $S: H \rightarrow Y$ such that $T=S R$

Proof. Since $\|S R x\| \leq\|S\|\|R x\|$, it is clear that (ii) implies (i). Assume now (i). Then there are Hilbert spaces $H_{1}, \ldots, H_{n}$ and $\sigma(X, F)$-to- $\sigma\left(H, H^{*}\right)$ continuous linear maps $R_{i}: X \rightarrow H_{i}$ such that $\|T x\| \leq \max _{1 \leq i \leq n}\left\|R_{i} x\right\|$ for all $x \in X$. Consider the $\ell^{2}$-direct sum $\tilde{H}=H_{1} \oplus \cdots \oplus H_{n}$, and define the linear map $R: X \rightarrow \tilde{H}$ by $R x=\left(R_{1} x, \ldots, R_{n} x\right)$. We regard $R$ as mapping $X$ into the closure $H$ of $T(X)$. It is easy to see that $T$ is $\sigma(X, F)$ to- $\sigma\left(H, H^{*}\right)$-continuous. Clearly, $\|T x\| \leq\|R x\|$ for all $x \in X$, so that we get a well-defined bounded linear map $\tilde{S}: R(X) \rightarrow Y$ satisfying $\tilde{S}(R x)=T x$ for all $x \in X$. Now define $S$ as the continuous extension of $S$ to $H$.

Corollary 2.2. The topology $s^{*}(X, F)$ is compatible with the duality $(X, F)$.

Proof. Suppose $f: X \rightarrow \mathbb{K}$ is a $s^{*}(X, F)$-continuous linear functional. The preceding result yields the existence of a Hilbert space $H$ with a $\sigma(X, F)$ to- $\sigma\left(H, H^{*}\right)$-continuous linear map $R: X \rightarrow H$ and a bounded (hence $\sigma\left(H, H^{*}\right)$-continuous) linear functional $g: H \rightarrow \mathbb{C}$ such that $f=g \circ R$. Thus $f$ is $\sigma(X, F)$-continuous. The converse is even more obvious.

In a special case the above corollary was also proved by a different method in [17, Corollary 9].

We omit the easy proof of the following result.

Proposition 2.3. Let $Z$ be a topological vector space. For a linear map $V: Z \rightarrow X$ the following conditions are equivalent: 
(i) $V$ is continuous when $X$ is equipped with the topology $s^{*}(X, F)$.

(ii) The composite map $R V$ is continuous from $Z$ to the norm topology of $H$ whenever $H$ is a Hilbert space and $R: X \rightarrow H$ is a $\sigma(X, F)$-to$\sigma\left(H, H^{*}\right)$-continuous linear map.

Remark 2.4. We have seen that the topology $s^{*}(X, F)$ has the following property: For any Banach space $Y$ and a linear map $T: X \rightarrow Y, T$ is $s^{*}(X, F)$-to-norm continuous if and only if $T$ has a factorization $T=$ $S R$ through a Hilbert space $H$ with $R: X \rightarrow H$ a $\sigma(X, F)$-to- $\sigma\left(H, H^{*}\right)$ continuous linear map and $S: H \rightarrow Y$ a bounded linear map. We are not claiming that $s^{*}(X, F)$ is the only such vector space topology, but it is certainly the weakest. Indeed, suppose that the topology $\tau$ makes $X$ into a topological vector space with just the property that whenever $H$ is a Hilbert space with a $\sigma(X, F)$-to- $\sigma\left(H, H^{*}\right)$-continuous linear map $R: X \rightarrow H$ and a bounded linear map $S: H \rightarrow Y$ into a Banach space $Y$, then $S R$ is $\tau$-tonorm continuous. Whenever $H$ is a Hilbert space, any $\sigma(X, F)$-to- $\sigma\left(H, H^{*}\right)$ continuous linear map may be composed with the identity map of $H$ and is therefore $\tau$-to-norm continuous. Applying the preceding proposition to the identity map on $X$ we thus see that $\tau$ is finer than $s^{*}(X, F)$.

We now consider an alternative, equivalent, approach to define $s^{*}(X, F)$ by means of sesquilinear forms rather than operators. The proof generalises an argument in the proof of [20, Corollary 1].

Proposition 2.5. For any mapping $\Gamma: X \times X \rightarrow \mathbb{K}$ the following conditions are equivalent:

(i) $\Gamma$ is a (conjugate-symmetric) sesquilinear form which is separately $\sigma(X, F)$-continuous and is positive (i.e., $\Gamma(x, x) \geq 0$ for all $x \in X)$.

(ii) There is a Hilbert space $H$ (with inner product $(\cdot \mid \cdot)$ ) and a $\sigma(X, F)-$ $\sigma\left(H, H^{*}\right)$-continuous linear map $T: X \rightarrow H$ such that

$$
\Gamma(x, y)=(T x \mid T y),
$$

for all $x, y \in X$.

In this situation $T$ is a bounded linear map and $\Gamma$ is jointly norm continuous.

Proof. The implication (ii) $\Longrightarrow$ (i) is immediate. Assume now (i). Denote $N=\{x \in X \mid \Gamma(x, x)=0\}$. The Cauchy-Schwarz inequality may be used to show that $N$ is a linear subspace of $X$, and there is a well-defined inner product on the quotient space $X / N$ such that $(x+N \mid y+N)=\Gamma(x, y)$ for all $x, y \in X$. Let $H$ be the Hilbert space completion of the inner product space $H_{0}=X / N$, and define $T x=x+N$. From the uniform boundedness principle it follows that $\Gamma$ is jointly norm continuous, and thus there is a constant $M>0$ such that $\|T x\|^{2}=\Gamma(x, x) \leq M\|x\|^{2}$, and so $T$ is a bounded linear map. We may identify $H$ with $H^{*}$ via the conjugate linear bijection $x \mapsto(\cdot \mid x)$. Since $\Gamma$ is separately $\sigma(X, F)$-continuous, $T$ is then $\sigma(X, F)-\sigma\left(H, H_{0}\right)$ continuous, and so its adjoint $T^{*}: H^{*} \rightarrow X^{*}$ maps $H_{0}$ 
into $E$. But since $T^{*}$ is norm continuous, it maps $H^{*}$, the closure of $H_{0}$, into $F$, and so $T$ is $\sigma(X, F)-\sigma\left(H, H^{*}\right)$-continuous.

In the sequel we only consider the cases $F=X^{*}$ or $F=X_{*}$ where $X_{*}$ is some Banach space having $X$ as its dual. (We use the notation $X_{*}$ for a predual of $X$, fixed in the given context, though usually $X$ need not have a unique predual.) Proposition 2.5 yields an alternative characterisation in terms of positive sesquilinear forms. In the case $E=X^{*}$ it is clearly equivalent to replace the $\sigma\left(X, X^{*}\right)$-to-weak continuity condition in the definition by the requirement of norm continuity. If $E=X_{*}$, we call $\sigma\left(X, X_{*}\right)$-to-weak continuity briefly weak*-continuity.

Using Corollary 2.2 we see that whenever $X$ and $Y$ are Banach spaces (respectively, dual Banach spaces) then a linear map $T: X \rightarrow Y$ is norm continuous or, equivalently, weakly continuous (respectively, weak*-weak*continuous) if and only if it is $s^{*}\left(X, X^{*}\right)-s^{*}\left(Y, Y^{*}\right)$ (respectively, $s^{*}\left(X, X_{*}\right)$ $\left.s^{*}\left(Y, Y_{*}\right)\right)$ continuous. (See the sixth point in Remark 5.9 for more details).

It is natural to ask whether $s^{*}(X)$ actually is the same as some well-known topology. Proposition 2.7 below rules out one candidate.

Lemma 2.6. Let $X$ be an infinite dimensional Banach space. Then there exists a bounded linear operator $T: X \rightarrow \ell_{2}$ with infinite dimensional range.

Proof. Let $\left\{\phi_{n}\right\}_{n \in \mathbb{N}}$ be a linearly independent bounded subset in $X^{*}$. The operator

$$
\begin{gathered}
T: X \rightarrow \ell_{2}, \\
T(x):=\left(n^{-1} \phi_{n}(x)\right)
\end{gathered}
$$

satisfies the claim.

Proposition 2.7. Let $X$ be a Banach space. If $s^{*}\left(X, X^{*}\right)$ coincides with the weak topology on $X$, then $X$ is finite dimensional.

Proof. Suppose that the $s^{*}\left(X, X^{*}\right)$-topology coincides with the weak topology on $X$. Let $T$ be a bounded linear operator from $X$ into a Hilbert space $H$. The set $\mathcal{O}:=\{x \in X:\|T(x)\|<1\}$ is a $s^{*}\left(X, X^{*}\right)$ neighbourhood of 0 , and hence, by hypothesis, a weak neighbourhood of 0 . Thus there exist $\phi_{1}, \ldots, \phi_{k} \in X^{*}$ such that

$$
\mathcal{O} \supset\left\{x \in X:\left|\phi_{j}(x)\right| \leq 1, \forall j=1, \ldots, k\right\} .
$$

Therefore, for each $x \in X$ it follows that

$$
\|T(x)\| \leq \sum_{j=1}^{k}\left|\phi_{j}(x)\right|,
$$

which implies that $\operatorname{ker}(T) \supset \cap_{j=1}^{k} \operatorname{ker}\left(\phi_{j}\right)$, and hence $T$ must have a finitedimensional range since $\operatorname{ker}(T)$ is finite codimensional. This shows that every bounded linear operator from $X$ into a Hilbert space has finite rank. Lemma 2.6 now gives the statement of the proposition. 
Because of the formal similarity of the definitions, many properties of the strong* topology have obvious analogues in the weak compactness right case. The proofs may be adapted with trivial changes, so we omit them. (Of course Remark 2.4 will have an obvious analogue, too.)

Proposition 2.8. Let $Y$ be a Banach space and $T: X \rightarrow Y$ a linear map. The following conditions are equivalent:

(i) $T$ is $\rho(X, F)$-to-norm continuous;

(ii) There exist a reflexive Banach space $E$ and a $\sigma(X, F)$-to- $\sigma\left(E, E^{*}\right)$ continuous linear map $R: X \rightarrow E$ and a bounded linear map $S: E \rightarrow Y$ such that $T=S R$.

Corollary 2.9. The topology $\rho(X, F)$ is compatible with the duality $(X, F)$.

Proposition 2.10. Let $Z$ be a topological vector space. For a linear map $V: Z \rightarrow X$ the following conditions are equivalent:

(i) $V$ is continuous when $X$ is equipped with the topology $\rho(X, F)$.

(ii) The composite map $R V$ is continuous from $Z$ to the norm topology of $E$ whenever $E$ is a reflexive space and $R: X \rightarrow E$ is a $\sigma(X, F)$-to$\sigma\left(E, E^{*}\right)$-continuous linear map.

In [17, Proposition 2, §4], it was shown that if $Y$ is a dual Banach space with a predual denoted by $Y_{*}$, then (in our present terminology) the topology $\rho\left(Y, Y_{*}\right)$ is the same as the Mackey topology $m\left(Y, Y_{*}\right)$. Now take $Y_{*}=X^{*}$ so that $Y=X^{* *}$. For a reflexive Banach space $E$ the $\sigma\left(X^{* *}, X^{*}\right)$-to- $\sigma\left(E, E^{*}\right)$ linear maps from $X$ into $E$ are, by restriction, in a bijective correspondence with the bounded linear maps from $X$ into $E$. From the quoted result of [17] it thus follows that $\rho(X)$, i.e. $\rho\left(X, X^{*}\right)$, is the same as the topology on $X$ induced by the Mackey topology $m\left(X^{* *}, X^{*}\right)$. As mentioned in the Introduction, this observation was published in [16, Proposition 2.2], and a different proof was given in [27, Corollary 4.2].

Since $F$ is a separating subspace of $X^{*}$, the mapping $x \mapsto \phi_{x}=\kappa(x)$ from $X$ into $F^{*}$ defined by $\phi_{x}(f)=f(x)$ is injective (though in general not isometric). We let $\tilde{\rho}(X, F)$ denote the topology of $X$ obtained when $X$ is identified with its image under the map $x \mapsto \phi_{x}$, and this image is given the topology induced by the Mackey topology $m\left(F, F^{*}\right)$. The next proposition generalises the result discussed in the preceding paragraph.

Proposition 2.11. The topologies $\rho(X, F)$ and $\tilde{\rho}(X, F)$ are the same.

Proof. Only a small modification to the case of $F=X^{*}$ is needed. Since for a reflexive Banach space $E$, the $\sigma(X, F)$-to- $\sigma\left(E, E^{*}\right)$-continuous linear maps $T: X \rightarrow E$ are in an obvious bijective correspondence with the $\sigma\left(F^{*}, F\right)$ to- $\sigma\left(E, E^{*}\right)$-continuous linear maps from $\kappa(X)$ to $F$, one may again use the same techniques applied in [17, Proposition $2, \S 4]$. 


\section{BACKGROUND MATERIAL ON JB*-TRIPLES AND JBW*-TRIPLES}

The strong* topology in the setting of $\mathrm{C}^{*}$-algebras and $\mathrm{JB}^{*}$-triples is a well-known tool, and it has been intensively developed especially during the last fifteen years. In this section we recall some of these developments.

Let us recall that a $J B^{*}$-triple is a complex Banach space $E$ equipped with a continuous triple product

$$
\begin{gathered}
\{., ., .\}: E \times E \times E \rightarrow E \\
(x, y, z) \mapsto\{x, y, z\}
\end{gathered}
$$

which is bilinear and symmetric in the outer variables and conjugate linear in the middle one and satisfies:

(a) (Jordan Identity)

$$
L(x, y)\{a, b, c\}=\{L(x, y) a, b, c\}-\{a, L(y, x) b, c\}+\{a, b, L(x, y) c\},
$$

for all $x, y, a, b, c \in E$, where $L(x, y): E \rightarrow E$ is the linear mapping given by $L(x, y) z=\{x, y, z\}$;

(b) The map $L(x, x)$ is an hermitian operator with non-negative spectrum for all $x \in E$;

(c) $\|\{x, x, x\}\|=\|x\|^{3}$ for all $x \in E$.

Every $\mathrm{C}^{*}$-algebra is a $\mathrm{JB}^{*}$-triple with respect to

$$
\{x, y, z\}:=2^{-1}\left(x y^{*} z+z y^{*} x\right),
$$

every $\mathrm{JB}^{*}$-algebra is a $\mathrm{JB}^{*}$-triple with triple product

$$
\{a, b, c\}=\left(a \circ b^{*}\right) \circ c+\left(c \circ b^{*}\right) \circ a-(a \circ c) \circ b^{*},
$$

and the Banach space $B(H, K)$ of all bounded linear operators between two complex Hilbert spaces $H, K$ is also an example of a $\mathrm{JB}^{*}$-triple with respect to $\{R, S, T\}=2^{-1}\left(R S^{*} T+T S^{*} R\right)$.

A $\mathrm{JBW}^{*}$-triple is a $\mathrm{JB}^{*}$-triple which is also a dual Banach space.

Given a $\mathrm{JB}^{*}$-triple $E$, a norm-one functional $\phi$ in $E^{*}$ and a norm-one element $e \in E^{* *}$ with $\phi(e)=1$, the mapping $x \mapsto\|x\|_{\phi}=\phi\{x, x, e\}^{\frac{1}{2}}$ defines a prehilbertian seminorm on $E$ which does not depend on the element $e$ (compare [4, Proposition 1.2]). By the classical little Grothendieck inequality for JB*-triples we know that when $E$ is a $\mathrm{JB}^{*}$-triple, then $s^{*}\left(E, E^{*}\right)$ coincides with the topology on $E$ generated by all the seminorms of the form $\|x\|_{\varphi}$, where $\varphi$ and $e$ are norm-one elements in $E^{*}$ and $E^{* *}$, respectively, and satisfy $\varphi(e)=1$ (see $[17, \S 4])$. Moreover, when $A$ is a $\mathrm{C}^{*}$-algebra, and $\phi$ is a positive functional in $A^{*}$, the mapping $(x, y) \mapsto \phi\left(x y^{*}+y^{*} x\right)$ also defines a positive sesquilinear form on $A \times A$. The classical algebra strong* topology of $A$ is the topology on $A$ generated by all the prehilbertian seminorms of the form

$$
\|x\|_{\phi}^{2}:=2^{-1} \phi\left(x x^{*}+x^{*} x\right) .
$$

It follows, as a direct consequence of the so-called little Grothendieck inequality (compare, [11], [19]), that the $s^{*}\left(A, A^{*}\right)$ topology coincides with the classical algebra strong* topology of $A$. 


\section{UNCONDITIONALLY CONVERGING AND QUASI COMPLETELY CONTINUOUS OPERATORS}

A series $\sum x_{n}$ in a Banach space is called weakly unconditionally Cauchy (w.u.C.) if there exists $C>0$ such that for any finite subset $F \subset \mathbb{N}$ and $\varepsilon_{n}= \pm 1$ we have $\left\|\sum_{n \in F} \varepsilon_{n} x_{n}\right\| \leq C$. We say that $\sum x_{n}$ is unconditionally convergent if any subseries is norm converging.

Since the $s^{*}(X)$-topology is coarser than $\rho(X)$, every $\rho(X)$-convergent net in $X$ is also $s^{*}(X)$-convergent to the same limit. In [18, Lemma 13] we proved that whenever $\sum_{n} x_{n}$ a w.u.C. series in a Banach space $X$, then $\left(x_{n}\right)$ is a $\rho(X)$-null sequence in $X$. These comments imply the following:

Lemma 4.1. Let $X$ be a Banach space and $\sum_{n} x_{n}$ a w.u.C. series in $X$. Then $\left(x_{n}\right)$ is an $s^{*}(X)$-null sequence in $X$.

Clearly, in general, a $s^{*}(X)$-null sequence in a Banach space $X$ need not define a w.u.C. series.

We recall here the following definition.

Definition 4.2. Let $X$ and $Y$ be Banach spaces and $T: X \rightarrow Y$ a linear mapping. We say that $T$ is unconditionally converging if for every w.u.C. series $\sum_{n} x_{n}$ in $X$, the series $\sum_{n} T\left(x_{n}\right)$ is unconditionally convergent.

Let us note for later use the following well-known fact: an operator $T$ : $X \longrightarrow Y$ is unconditionally converging if and only if for every w.u.C. series $\sum_{n} x_{n}$ in $X$, we have $\left\|T\left(x_{n}\right)\right\| \rightarrow 0$. One of the implications is very easy. For the other implication it is enough to recall that, if $T$ is not unconditionally converging, then $T$ "fixes a copy of $c_{0}$ " (see [8, Exercise V.8]); that is, there exists a w.u.C. series $\sum x_{n} \subset X$, such that the sequence $\left(T\left(x_{n}\right)\right)_{n}$ is equivalent to the usual $c_{0}$ basis.

Let us also recall that a Banach space $X$ is said to have Pełczyński's property $(\mathrm{V})$ if, for every Banach space $Y$, every unconditionally converging operator is weakly compact.

The following definition is inspired by the concept of quasi completely continuous operators from a $\mathrm{C}^{*}$-algebra to a Banach space given by the third and the fourth authors of this note in [28, Definiton 2.1]. We shall see in Corollary 5.10 that this definition is in fact equivalent, in the setting of $\mathrm{C}^{*}$-algebras, to the one introduced in the just quoted paper.

Definition 4.3. Let $X$ and $Y$ be Banach spaces and $T: X \rightarrow Y$ a linear mapping. We say that $T$ is quasi completely continuous (QCC) if for every $s^{*}(X)$ Cauchy sequence $x_{n}$ in $X$, the sequence $\left(T\left(x_{n}\right)\right)$ is norm convergent.

We recall that a bounded linear operator $T$ between two Banach spaces is called completely continuous if it maps weakly Cauchy sequences into norm convergent sequences.

We shall now give a counterexample showing that not every unconditionally converging operator is QCC. (A different approach will be used later in Remark 5.12.) We recall that a Banach space $X$ is said to be an $\mathcal{L}_{\infty}$-space 
if there exists $\lambda \geq 1$ such that every finite dimensional subspace $M \subset X$ is contained in a finite dimensional space $F \subset X$ for which there exists an isomorphism $T: F \rightarrow \ell_{\infty}^{\operatorname{dim}(F)}$ such that $\|T\|\left\|T^{-1}\right\|<\lambda$. Let $X$ be an $\mathcal{L}_{\infty}$-space without copies of $c_{0}$ and with a copy of $\ell_{2}$ (the existence of such objects follows from [5]). Consider the identity in $X$. Since $X$ does not contain $c_{0}$, the identity in $X$ is unconditionally converging. To see that it is not QCC we first need a simple Lemma.

Let $1 \leq p \leq \infty$. A sequence $\left(x_{n}\right)$ in a Banach space $Y$ is said to be weakly $p$-summable if, for every $\phi \in Y^{*}$, the scalar sequence $\left(\left\langle\phi, x_{n}\right\rangle\right)$ is in $\ell_{p}$. In that case we define its $p$-weak norm by

$$
\left\|\left(x_{n}\right)\right\|_{p}^{\omega}=\sup _{\|\phi\| \leq 1}\left(\sum_{n}\left|\left\langle\phi, x_{n}\right\rangle\right|^{p}\right)^{1 / p} .
$$

It is well known (and easy to prove) that $\left(x_{n}\right)$ is weakly $p$-summable if and only if the operator $\ell_{p^{\prime}} \longrightarrow Y$ sending $e_{n}=\left(\delta_{n k}\right)_{k \in \mathbb{N}}$ to $x_{n}$ is bounded (here $p^{\prime}$ denotes the conjugate of $p$, that is, $1 / p^{\prime}+1 / p=1$; if $p=1$ then $c_{0}$ should replace $\left.\ell_{\infty}\right)$. In fact the norm of that operator equals $\left\|\left(x_{n}\right)\right\|_{p}^{\omega}$.

We recall that a bounded linear operator $T$ between two Banach spaces, $X$ and $Y$, is $p$-summing if it takes weakly $p$-summable sequences into $p$ summable sequences; in that case there exists a constant $K>0$ satisfying

$$
\left(\sum_{n=1}^{\infty}\left\|T\left(x_{n}\right)\right\|^{p}\right)^{\frac{1}{p}} \leq K\left\|\left(x_{n}\right)\right\|_{p}^{w},
$$

for every weakly $p$-summable sequence $\left(x_{n}\right)$ in $X$.

Lemma 4.4. If $X$ is an $\mathcal{L}_{\infty}$-space, every weakly 2-summable sequence $\left(x_{n}\right)$ in $X$ is $s^{*}(X)$-null.

Proof. Let $T: X \longrightarrow \ell_{2}$ be a bounded linear operator. By Grothendieck's inequality, $T$ is 2-summing, and hence $\sum_{n}\left\|T\left(x_{n}\right)\right\|^{2} \leq K\left\|\left(x_{n}\right)\right\|_{2}^{w}<\infty$. In particular $T\left(x_{n}\right)$ is norm-null.

Let again $X$ be an $\mathcal{L}_{\infty}$-space without copies of $c_{0}$ and with a copy of $\ell_{2}$. We now consider $\left(x_{n}\right) \subset X$, a sequence equivalent to the usual $\ell_{2}$ basis (such a sequence exists since $X$ contains a copy of $\ell_{2}$ ). By the preceding lemma, $\left(x_{n}\right)$ is strong*-null, but clearly it is not norm-null, which proves that the identity in $X$ is not QCC.

Remark 4.5. Let $X$ be a Banach space satisfying the Dunford-Pettis property (DPP). Then the topologies $s^{*}\left(X, X^{*}\right)$ and $\rho(X)$ on $X$ have the same convergent sequences. Indeed, let $\left(x_{n}\right)$ be a (bounded) $s^{*}\left(X, X^{*}\right)$-convergent sequence in $X$ with limit $x_{0}$, and let $T: X \rightarrow R$ be a bounded linear operator from $X$ into a reflexive Banach space $R$. Since $T$ is weakly compact and $X$ has DPP, then $T$ is completely continuous, and hence $T\left(x_{n}-x_{0}\right)$ tends to zero in norm. This shows that $\left(x_{n}\right)$ tends to $x_{0}$ in the $\rho(X)$-topology. 
Remark 4.6. We would like to finish this series of remarks by showing that the $\mathrm{s}^{*}\left(X, X^{*}\right)$ topology does not coincide, in general, with the weak nor weak* topology. Consider, for example $\ell_{p}$ with $1 \leq p<2$. Since the natural inclusion of $\ell_{p}$ into $\ell_{2}$ is contractive, we deduce that the natural basis of $\ell_{p}$ is a weakly-null sequence which is never $s^{*}\left(\ell_{p}, \ell_{p}^{*}\right)$-null (compare also Proposition 2.7.)

\section{When do the topogies $s^{*}(X)$ And $\rho(X)$ AGREe on Bounded SUBSETS OF $X$ ?}

Our next step is to investigate the connections between the strong*, Mackey and w-right topologies. By [17, Proposition 2, §4], we know that the Mackey topology $m\left(Y, Y_{*}\right)$ coincides with the topology on $Y$ generated by all the seminorms $\||\cdot|\|_{T}$, where $T$ is a weak*-continuous linear operator from $Y$ into a reflexive Banach space.

The next result was proved in [17, Proposition 3].

Proposition 5.1. Let $Y$ be a dual Banach space with a predual denoted by $Y_{*}$. The following assertions are equivalent:

a) The topologies $m\left(Y, Y_{*}\right)$ and $s^{*}\left(Y, Y_{*}\right)$ coincide on bounded subsets of $Y$.

b) For every weak*-continuous linear operator $F$ from $Y$ into a reflexive Banach space, there exists a weak*-continuous linear operator $G$ from $Y$ to a Hilbert space satisfying $\|F(x)\| \leq\|G(x)\|+\|x\|$ for all $x \in Y$.

c) For every weak ${ }^{*}$-continuous linear operator $F$ from $Y$ into a reflexive Banach space, there exist a weak*-continuous linear operator $G$ from $Y$ to a Hilbert space and a mapping $N:(0, \infty) \rightarrow(0, \infty)$ satisfying

$$
\|F(x)\| \leq N(\varepsilon)\|G(x)\|+\varepsilon\|x\|
$$

for all $x \in Y$ and $\varepsilon>0$.

From [17, page 621] (respectively, [1, Theorem II.7]) it follows that when $W$ is a $\mathrm{JBW}^{*}$-triple (respectively, a von Neumann algebra) then the topologies $m\left(W, W_{*}\right)$ and $s^{*}\left(W, W_{*}\right)$ coincide on bounded subsets of $W$. When $\mathrm{JBW}^{*}$-triples and von Neumann algebras are replaced with $\mathrm{JB}^{*}$-triples and $\mathrm{C}^{*}$-algebras, respectively, then the strong*-topology and the w-right topology coincide on the unit ball. The following proposition shows that an analogous result is true for general Banach spaces. We should note that statements $b$ ) and $c$ ) in the following proposition were proved when $X$ is a $\mathrm{C}^{*}$-algebra in [13].

Proposition 5.2. Let $X$ be a Banach space. Then the following assertions are equivalent:

a) The w-right topology and the $s^{*}\left(X, X^{*}\right)$-topology coincide on bounded subsets of $X$. 
b) The topologies $m\left(X^{* *}, X^{*}\right)$ and $s^{*}\left(X^{* *}, X^{*}\right)$ coincide on bounded subsets of $X^{* *}$.

c) For every bounded linear operator $F$ from $X$ to a reflexive Banach space, there exist a bounded linear operator $G$ from $X$ to a Hilbert space and a mapping $N:(0, \infty) \rightarrow(0, \infty)$ satisfying

$$
\|F(x)\| \leq N(\varepsilon)\|G(x)\|+\varepsilon\|x\|,
$$

for all $x \in X$ and $\varepsilon>0$.

d) For every bounded linear operator $F$ from $X$ to a reflexive Banach space, there exists a bounded linear operator $G$ from $X$ to a Hilbert space satisfying $\|F(x)\| \leq\|G(x)\|+\|x\|$, for all $x \in X$.

Proof. $a) \Rightarrow b)$ As we have already commented before, every $s^{*}\left(X^{* *}, X^{*}\right)$ neighbourhood of 0 in $X^{* *}$ is also a $m\left(X^{* *}, X^{*}\right)$-neighbourhood of 0 in $X^{* *}$. To see the converse inclusion, let $\mathcal{O}^{\prime}$ be a relative $m\left(X^{* *}, X^{*}\right)$-neighbourhood of 0 in $B_{X^{* *}}$. As we have commented before, from [17, Proposition 2 ] it follows that $m\left(X^{* *}, X^{*}\right)$ coincides with the topology on $X^{* *}$ generated by all the seminorms $x \mapsto\|T(x)\|$, where $T$ is a weak*-to-weak continuous linear operator from $X^{* *}$ into a reflexive Banach space. We may therefore assume that $\mathcal{O}^{\prime}$ is of the form

$$
\mathcal{O}^{\prime}=\left\{x \in B_{X^{* *}}:\|T(x)\| \leq \delta\right\},
$$

where $T: X^{* *} \rightarrow R$ is a weak* continuous linear operator from $X^{* *}$ into a reflexive Banach space and $\delta$ is a positive constant. Thus, there exists $S: R^{*} \rightarrow X^{*}$ satisfying $s^{*}=T$. It can be easily checked that $S$ is $\sigma\left(R^{*}, R\right)$ to- $\sigma\left(X^{*}, X\right)$-continuous. In particular, we can find a bounded linear operator $U: X \rightarrow R$ such that $U^{* *}=T$. The set

$$
\mathcal{O}=\left\{x \in B_{X}:\|U(x)\| \leq \delta\right\}
$$

is a relative w-right-neighbourhood of 0 in $B_{X}$. Since, by hypothesis, the w-right topology and the $s^{*}\left(X, X^{*}\right)$-topology coincide on bounded subsets of $X$, there exist bounded linear operators $G_{1}, \ldots, G_{k}$, from $X$ into Hilbert spaces $H_{1}, \ldots, H_{k}$, such that

$$
\mathcal{O} \supseteq\left\{x \in B_{X}:\left\|G_{i}(x)\right\| \leq \delta ; \forall 1 \leq i \leq k\right\} .
$$

Clearly, $G_{i}^{* *}: X^{* *} \rightarrow H_{i}$ is a weak*-continuous operator. We claim that

$$
\mathcal{O}^{\prime} \supseteq\left\{x \in B_{X^{* *}}:\left\|G_{i}^{* *}(x)\right\| \leq \frac{\delta}{2} ; \forall 1 \leq i \leq k\right\} .
$$

Indeed, let $z$ be an element in the right hand set. Since the $s^{*}\left(X^{* *}, X^{*}\right)$ topology is compatible with the duality $\left(X^{* *}, X^{*}\right)$, then, by Goldstine's and bipolar theorems, there exists a net $\left(x_{\lambda}\right)$ in $B_{X}$ converging to $z$ in the $s^{*}\left(X^{* *}, X^{*}\right)$ topology of $X^{* *}$. Thus, for each $1 \leq i \leq k$,

$$
\left\|G_{i}\left(x_{\lambda}\right)\right\|=\left\|G_{i}^{* *}\left(x_{\lambda}\right)\right\| \rightarrow\left\|G_{i}^{* *}(z)\right\| \leq \frac{\delta}{2} .
$$


Therefore, there exists $\lambda_{0}$ such that for each $\lambda \geq \lambda_{0}$, we have $\left\|G_{i}\left(x_{\lambda}\right)\right\|<\delta$, for all $1 \leq i \leq k$, which shows, by (1), that $x_{\lambda} \in \mathcal{O}$, equivalently, $\left\|U\left(x_{\lambda}\right)\right\| \leq$ $\delta$, for each $\lambda \geq \lambda_{0}$.

Since the net $\left(x_{\lambda}\right)_{\lambda \geq \lambda_{0}}$ also converges in the weak* topology of $X^{* *}$ to $z$, and the norm is weak* lower semi-continuous, we have

$$
\|T(z)\| \leq \liminf _{\lambda \geq \lambda_{0}}\left\|T\left(x_{\lambda}\right)\right\|=\liminf _{\lambda \geq \lambda_{0}}\left\|U^{* *}\left(x_{\lambda}\right)\right\|=\liminf _{\lambda \geq \lambda_{0}}\left\|U\left(x_{\lambda}\right)\right\| \leq \delta .
$$

This proves that $z \in \mathcal{O}^{\prime}$, and the claim. Therefore $\mathcal{O}^{\prime}$ contains the set $\left\{x \in B_{X^{* *}}:\left\|G_{i}^{* *}(x)\right\| \leq \frac{\delta}{2} ; \forall 1 \leq i \leq k\right\}$, and hence $\mathcal{O}^{\prime}$ is a relative $s^{*}\left(X^{* *}, X^{*}\right)$-neighbourhood of 0 in $B_{X^{* *}}$.

$b) \Rightarrow c)$ Let $F: X \rightarrow R$ and $G: X^{* *} \rightarrow H$ be bounded linear operators from $X$ and $X^{* *}$ into a reflexive Banach space and a Hilbert space, respectively. Suppose that $G$ is weak*-continuous. Since $F^{* *}: X^{* *} \rightarrow R$ is a weak*-continuous linear operator and there exists a bounded linear operator $U: X \rightarrow H$ satisfying $U^{* *}=G$, then statement $c$ ) follows from $b$ ) via Proposition 5.1.

The implication $c) \Rightarrow d$ ) is clear.

$d) \Rightarrow b$ ) Let $T: X^{* *} \rightarrow R$ be a weak* continuous linear operator from $X^{* *}$ into a reflexive Banach space. According to what we have seen in the proof of $a) \Rightarrow b$ ), there exists a bounded linear operator $U: X \rightarrow R$ satisfying $U^{* *}=T$. By hypothesis, there exists a bounded linear operator $G$ from $X$ to a Hilbert space, $H$, satisfying

$$
\|U(x)\| \leq\|G(x)\|+\|x\|,
$$

for all $x \in X$. Clearly $G^{* *}: X^{* *} \rightarrow H$ is a weak* continuous operator. Let $z \in X^{* *} \backslash\{0\}$. Since the $s^{*}\left(X^{* *}, X^{*}\right)$-topology is compatible with the duality $\left(X^{* *}, X^{*}\right)$, there exists a net $\left(x_{\lambda}\right)$ in $\|z\| B_{X}$ converging to $z$ in the $s^{*}\left(X^{* *}, X^{*}\right)$ topology of $X^{* *}$. Thus,

$$
\left\|G\left(x_{\lambda}\right)\right\|=\left\|G^{* *}\left(x_{\lambda}\right)\right\| \rightarrow\left\|G^{* *}(z)\right\| .
$$

Since the net $\left(x_{\lambda}\right)$ also converges in the weak ${ }^{*}$ topology of $X^{* *}$ to $z$, and the norm is weak* lower semi-continuous, we have

$$
\|T(z)\| \leq \liminf \left\|T\left(x_{\lambda}\right)\right\|=\liminf \left\|U^{* *}\left(x_{\lambda}\right)\right\|=\liminf \left\|U\left(x_{\lambda}\right)\right\| .
$$

Finally, we deduce from (3) and (4) that

$$
\|T(z)\| \leq\left\|G^{* *}(z)\right\|+\|z\|,
$$

for all $z \in X^{* *}$, which implies, by Proposition 5.1, that the topologies $m\left(X^{* *}, X^{*}\right)$ and $s^{*}\left(X^{* *}, X^{*}\right)$ coincide on bounded subsets of $X^{* *}$.

Finally, the implication $b) \Rightarrow a$ ) is clear.

Remark 5.3. We have already seen that when $W$ is a $\mathrm{JBW}^{*}$-triple or a von Neumann algebra then the topologies $m\left(W, W_{*}\right)$ and $s^{*}\left(W, W_{*}\right)$ coincide on bounded subsets of $W$ (see [1, Theorem II.7] and [17, page 621], 
respectively). Since, by [9], the bidual of every $\mathrm{JB}^{*}$-triple (respectively, $\mathrm{C}^{*}$ algebra) is a $\mathrm{JBW}^{*}$-triple (respectively, a von Neumann algebra), Proposition 5.2 guarantees that for every $\mathrm{JB}^{*}$-triple, $E$, the w-right topology and $s^{*}\left(E, E^{*}\right)$ topology coincide on bounded subsets of $E$. In particular, this is true when $E$ is a $\mathrm{C}^{*}$-algebra.

The next lemma gives sufficient conditions to guarantee that, in a Banach space $X$, the $s^{*}(X)$-topology and the $\sigma\left(X, X^{*}\right)$-topology coincide on bounded sets of $X$.

Lemma 5.4. Let $X$ be a Banach space. Suppose that every bounded conjugate linear operator $T$ from $X$ to $X^{*}$ is compact. Then the $s^{*}(X)$ topology coincides with the $\sigma\left(X, X^{*}\right)$ topology on bounded sets of $X$.

Proof. We have already seen that the $s^{*}(X)$-topology is compatible with the duality $\left(X, X^{*}\right)$. Therefore every $s^{*}(X)$-null net in $X$ is automatically weakly-null. To see the other implication, let $\left(x_{\lambda}\right)$ be a bounded weakly-null net in $X$. We may assume that $\left\|x_{\lambda}\right\| \leq 1$, for all $\lambda$.

Let $\Gamma: X \times X \rightarrow \mathbb{K}$ be a (norm) continuous positive sesquilinear form on $X$. Let $T: X \rightarrow X^{*}$ be the bounded conjugate-linear operator defined by $T(y)(x):=\Gamma(x, y),(x, y \in X)$. By hypothesis, $T$ is compact.

Denote $N=\{x \in X \mid \Gamma(x, x)=0\}$. As we have seen before, the CauchySchwarz inequality may be used to show that $N$ is a linear subspace of $X$, and there is a well-defined inner product on the quotient space $X / N$ such that $(x+N \mid y+N)=\Gamma(x, y)$ for all $x, y \in X$. Let $H$ denote the Hilbert space completion of the inner product space $H_{0}=X / N$. The operator $R: X \rightarrow H$, $R(x):=x+N$ is continuous. We claim that $R$ is compact. Indeed, let $\left(y_{n}\right)$ be a sequence in the closed unit ball of $X$. Since $T$ is compact, there exists a subsequence $\left(y_{\sigma(n)}\right)$ such that $T\left(y_{\sigma(n)}\right)$ is norm convergent. The formula

$$
\begin{gathered}
\left\|R\left(y_{\sigma(n)}\right)-R\left(y_{\sigma(m)}\right)\right\|^{2}=\left\|y_{\sigma(n)}-y_{\sigma(m)}+N\right\|^{2} \\
=\Gamma\left(y_{\sigma(n)}-y_{\sigma(n)}, y_{\sigma(n)}-y_{\sigma(m)}\right) \leq\left\|T\left(y_{\sigma(n)}-y_{\sigma(m)}\right)\right\|\left\|y_{\sigma(n)}-y_{\sigma(m)}\right\| \\
\leq 2\left\|T\left(y_{\sigma(n)}\right)-T\left(y_{\sigma(m)}\right)\right\|,
\end{gathered}
$$

shows that $\left(R\left(y_{\sigma(n)}\right)\right)$ is a Cauchy sequence in $H$, and hence $R$ is compact.

Since every Hilbert space has the approximation property, given $\varepsilon>0$, there exists a finite rank operator $F: X \rightarrow H$, such that $\|R-F\|<\varepsilon / 2$. Since $F$ has finite rank and $\left(x_{\lambda}\right)$ is weakly-null, there exists $\lambda_{0}$ such that for every $\lambda \geq \lambda_{0}$ we have $\left\|F\left(x_{\lambda}\right)\right\|<\varepsilon / 2$. Finally, for each $\lambda \geq \lambda_{0}$ it follows that

$$
\left\|R\left(x_{\lambda}\right)\right\| \leq\left\|(R-F)\left(x_{\lambda}\right)\right\|+\left\|F\left(x_{\lambda}\right)\right\|<\varepsilon,
$$

which implies that $\sqrt{\Gamma\left(x_{\lambda}, x_{\lambda}\right)}=\left\|x_{\lambda}+N\right\|=\left\|R\left(x_{\lambda}\right)\right\|_{\lambda} 0$. This implies that, for each $\Gamma$ as above, we have $\Gamma\left(x_{\lambda}, x_{\lambda}\right) \rightarrow 0$. Since the $s^{*}(X)$ topology is defined by all the continuous positive sesquilinear forms $\Gamma$ on $X \times X$, we deduce that $\left(x_{\lambda}\right)$ also is $s^{*}\left(X, X^{*}\right)$-null. 
Let $X$ and $Y$ be two complex Banach spaces such that every linear operator $T: X \rightarrow Y$ is compact. If there exists a conjugate-linear isomorphism $J: Y \rightarrow Y$, then every conjugate-linear operator $R: X \rightarrow Y$ is also compact.

Let $2<p<+\infty$. We have seen in the above remark that on $\ell_{p}$ weakly-null and $s^{*}\left(\ell_{p}, \ell_{p}^{*}\right)$-null sequences coincide. We can now see that both topologies are in fact the same on bounded sets. Denote $\ell_{p}^{*}=\ell_{q}$, for a suitable $q$. In this case we have $q<p$ and hence, by Pitt's Theorem, every bounded linear operator $T: \ell_{p} \rightarrow \ell_{p}^{*}=\ell_{q}$ (and every $T: c_{0} \rightarrow c_{0}^{*}=\ell_{1}$ ) is compact. This fact together with the above lemma and comments show that, for each $2<p<+\infty, s^{*}\left(\ell_{p}, \ell_{p}^{*}\right)$ coincides with $\sigma\left(\ell_{p}, \ell_{p}^{*}\right)$ (respectively, $s^{*}\left(c_{0}, c_{0}^{*}\right)$ coincides with $\left.\sigma\left(c_{0}, c_{0}^{*}\right)\right)$ on bounded sets of $\ell_{p}$ (respectively, $c_{0}$ ).

The strong* topology in a von Neumann algebra or in a $\mathrm{JBW}^{*}$-triple has proved to be a very good tool to characterise relatively weakly compact subsets in their respective preduals (see for example [24], [1], [2], [22], and [15]). The following proposition generalises the above cited results to the class of Banach spaces $Y$ in which the topologies $s^{*}\left(Y^{* *}, Y^{*}\right)$ and $\rho(Y)$ coincide on bounded subsets; the proof is very similar to the one given in [15, Theorem 2.1].

Proposition 5.5. Let $X$ be a Banach space such that the $s^{*}\left(X, X^{*}\right)$ and $w$-right topologies coincide on bounded subsets of $X$, and let $K$ be a relatively weakly compact subset in $X^{*}$. Then there exists a bounded linear operator $R$ from $X$ into a Hilbert space with the following property: for every $\varepsilon>0$ there exists $\delta>0$ such that for each $x$ in $X$ with $\|x\| \leq 1$, and $\|R(x)\|<\delta$, we have $|\varphi(x)|<\varepsilon$, for all $\varphi \in K$.

Proof. Let $D=\overline{\mid c o}^{w}(K)$ be the weakly-closed absolutely convex hull of $K$ in $X^{*}$. Let us define a bounded linear operator

$$
T: \ell_{1}(D) \rightarrow X^{*}
$$

given by

$$
T\left(\left\{\lambda_{\varphi}\right\}_{\varphi \in D}\right):=\sum_{\varphi \in D} \lambda_{\varphi} \varphi
$$

Since $T\left(B_{\ell_{1}(D)}\right)=D$ is weakly compact, then $T$ (and hence $T^{*}$ ) is a weakly compact operator. By Proposition 5.2 it follows that $s^{*}\left(X^{* *}, X^{*}\right)$ and $m\left(X^{* *}, X^{*}\right)$ coincide on bounded subsets of $X^{* *}$. Now applying [17, Proposition 3] to $T^{*}$ we guarantee the existence of a weak* continuous linear operator $R$ from $X^{* *}$ to a Hilbert space $H$ and a mapping $N:(0,+\infty) \rightarrow(0,+\infty)$ satisfying

$$
\|T(z)\| \leq N(\varepsilon)\|R(z)\|+\varepsilon / 2\|z\|,
$$

for all $z \in X^{* *}, \varepsilon>0$. As we have seen several times before, we can regard $R$ as the bitranspose of a bounded linear operator from $X$ to $H$, which we shall continue to denote by $R$. Since for each $x \in X$ we have

$$
\sup _{\varphi \in K}|\phi(x)|=\sup _{\varphi \in D}|\phi(x)|=\sup _{y \in B_{\ell_{2}(D)}}|T(y)(x)|=\sup _{y \in B_{\ell_{2}(D)}}\left|T^{*}(x)(y)\right|
$$




$$
\leq\left\|T^{*}(x)\right\| \leq N(\varepsilon)\|R(x)\|+\varepsilon / 2\|x\|,
$$

for all $\varepsilon>0$, we can easily deduce the statement of the proposition.

When we assume property $(V)$ for the space $X$, then we obtain a converse of the above result.

Proposition 5.6. Let $X$ be a Banach space satisfying property $(V)$ and such that the $s^{*}\left(X, X^{*}\right)$ and the w-right topologies coincide on bounded subsets of $X$. Let $K$ be a bounded subset in $X^{*}$. Then $K$ is relatively weakly compact if and only if there exists a bounded linear operator $R$ from $X$ into a Hilbert space with the following property: for every $\varepsilon>0$ there exists $\delta>0$ such that for each $x \in X$ with $\|x\| \leq 1$ and $\|R(x)\|<\delta$ we have $|\varphi(x)|<\varepsilon$, for all $\varphi \in K$.

Proof. The "only if" implication follows from Proposition 5.5 above. Suppose now that there exists a bounded linear operator $R$ from $X$ into a Hilbert space satisfying that

for each $\varepsilon>0$, there exists $\delta>0$ such that for all $x \in X$, with

(5) $\|x\| \leq 1$, and $\|R(x)\|<\varepsilon$ we have $|\varphi(x)|<\varepsilon$, for every $\varphi \in K$.

Let $\sum_{n} x_{n}$ be a w.u.C. series in $X$. By Lemma 4.1, it follows that $\left(x_{n}\right)$ is a $s^{*}(X)$-null sequence in $X$, thus

$$
\left\|R\left(x_{n}\right)\right\|_{n \rightarrow+\infty} 0 .
$$

Therefore, inequality (5) gives that

$$
\lim _{n \rightarrow+\infty} \sup _{\varphi \in K}\left|\varphi\left(x_{n}\right)\right|=0 .
$$

Finally, since $X$ has property $(V)$ we deduce that $K$ is relatively weakly compact.

To prove the "if" implication of the above proposition we simply need property $(V)$ on $X$, the other hypothesis is only used in the "only if" implication.

For the class of Banach spaces $X$ in which the w-right topology and the $s^{*}\left(X, X^{*}\right)$ topology coincide on bounded sets we have:

Theorem 5.7. Let $X$ and $Y$ be Banach spaces and $T: X \rightarrow Y$ a linear mapping. Suppose that the w-right and the $s^{*}\left(X, X^{*}\right)$ topologies coincide on bounded sets of $X$ and $X$ has property $(V)$. Then the following assertions are equivalent:

a) $T$ is unconditionally converging.

b) $T$ is weakly compact.

c) There exist a bounded linear operator $G$ from $X$ into a Hilbert space and a mapping $N:(0, \infty) \rightarrow(0, \infty)$ satisfying

$$
\left\|T^{* *}(x)\right\| \leq N(\varepsilon)\left\|G^{* *}(x)\right\|+\varepsilon\|x\|
$$

for all $x \in X^{* *}$ and $\varepsilon>0$. 
d) If $\left(x_{\lambda}\right)$ is a bounded net in $X^{* *}$ converging to some element $x \in X^{* *}$ in the $s^{*}\left(X^{* *}, X^{*}\right)$ topology, then, for each $\lambda, T^{* *}\left(x_{\lambda}\right)$ and $T^{* *}(x)$ are in $Y$ and $\left\|T^{* *}\left(x_{\lambda}\right)-T^{* *}(x)\right\|$ converges to 0 .

e) $T$ is $Q C C$.

Proof. We recall first that, since in every Banach space $X$, the norm topology is always stronger than the $s^{*}\left(X, X^{*}\right)$ topology, every quasi completely continuous mapping from $X$ to a Banach space is always norm continuous.

$a) \Rightarrow b)$ follows by property $(V)$ on $X$.

The equivalence $b) \Leftrightarrow c$ ) follows from Proposition 5.2.

$c) \Rightarrow d)$ and $d) \Rightarrow e$ ) are clear.

$e) \Rightarrow a)$ Let $\sum x_{n}$ be a w.u.C. series in $X$. Since by Lemma 4.1, we have $\left(x_{n}\right) \rightarrow 0$ in the $s^{*}(X)$ topology, we deduce by hypothesis that $\left\|T\left(x_{n}\right)\right\| \rightarrow 0$, which shows that $T$ is unconditionally converging by the remarks preceding this Theorem.

Remark 5.8. In [18, Theorem 4], it is shown that for a linear operator $T$ between two Banach spaces $X$ and $Y, T$ is weakly compact if, and only if, $T$ is continuous from $B_{X}$ (the unit ball) equipped with the right Topology, into $Y$, equipped with the norm topology. The latter statement could also be added to the list of equivalences given in the above theorem.

Remark 5.9. - Note that the implication e) $\Rightarrow$ a) above did not use any assumptions on $X, Y$. That is, every QCC linear operator is unconditionally converging.

- There are unconditionally converging operators not quasi completely continuous, see Remark 5.12.

- Every completely continuous operator is QCC. This follows easily from the trivial fact that every $s^{*}\left(X, X^{*}\right)$-null sequence is clearly weakly-null.

- There exist QCC operators which are not completely continuous: Consider for example the identity in $\ell_{2}$. Related to this, we observe that when $X=\ell_{2}$ we find an analogue of Schur's Lemma for the $s^{*}\left(X, X^{*}\right)$ topology: If a net in $\ell_{2}$ is strong*-null, then it is also norm-null.

- Not every QCC operator is weakly compact: take any completely continuous not weakly compact operator, for example the identity in $\ell_{1}$. Related to this, it seems interesting to study the class of spaces $X$ such that, for every Banach space $Y$, every QCC operator $T: X \longrightarrow Y$ is weakly compact. We shall discuss this in the last section.

- Let $X, Y$ and $Z$ be Banach spaces. If $T \in L(X, Y)$ is QCC and $R: Y \rightarrow Z$ and $U: Z \rightarrow X$ are bounded linear operators, then $R T$ and $T U$ are both QCC. To see the latter, we simply observe that every $U$ in $L(Z, X)$ is $s^{*}\left(Z, Z^{*}\right)-s^{*}\left(X, X^{*}\right)$ continuous. Indeed, for each Banach space $B$, $s^{*}\left(B, B^{*}\right)$ is the topology generated by all continuous positive sesquilinear forms $\Gamma: B \times B \rightarrow \mathbb{K}$ (see Proposition 2.5). Given a continuous positive 
sesquilinear form $\Gamma$ on $X \times X$, the mapping $\left(z_{1}, z_{2}\right) \mapsto \Gamma\left(U\left(z_{1}\right), U\left(z_{2}\right)\right)$ also defines a continuous positive sesquilinear form on $Z \times Z$. This implies that $U$ is $s^{*}\left(Z, Z^{*}\right)-s^{*}\left(X, X^{*}\right)$ continuous. In fact, we have that $U$ is in $L(Z, X)$ if, and only if, $U$ is $s^{*}\left(Z, Z^{*}\right)-s^{*}\left(X, X^{*}\right)$ continuous, since the $s^{*}\left(B, B^{*}\right)$ topology on a Banach space, $B$, is compatible with the duality $\left(B, B^{*}\right)$.

For every $\mathrm{JB}^{*}$-triple $E$, the right and $s^{*}\left(E, E^{*}\right)$ topologies coincide on bounded subsets of $E$ (see Remark 5.3). Since every JB*-triple also satisfies property $(V)$ (see $[6]$ ), the next corollary now follows as a consequence of Theorem 5.7.

Given two norm-one continuous linear functionals, $\varphi_{1}$ and $\varphi_{2}$, in the dual space of a JB*-triple $E,\|\cdot\|_{\varphi_{1}, \varphi_{2}}$ will stand for the prehilbertian seminorm on $E$ defined by $\|\cdot\|_{\varphi_{1}, \varphi_{2}}:=\sqrt{\|\cdot\|_{\varphi_{1}}^{2}+\|\cdot\|_{\varphi_{2}}^{2}}$.

Corollary 5.10. Let $E$ be a JB*-triple, $X$ a Banach space and $T: E \rightarrow X$ a linear mapping. Then all the statements in Theorem 5.7 are equivalent for $T$. In this particular setting they are also equivalent to the following:

$\left.c^{\prime}\right)$ There exist a mapping $N:(0,+\infty) \rightarrow(0,+\infty)$ and $\varphi_{1}, \varphi_{2}$ in the unit sphere of $E^{*}$ such that for each $\varepsilon>0$ and $\alpha \in E^{* *}$ we have

$$
\left\|T^{* *}(\alpha)\right\| \leq N(\varepsilon)\|\alpha\|_{\varphi_{1}, \varphi_{2}}+\varepsilon\|\alpha\|
$$

To see the equivalence with " $\mathrm{c}^{\prime}$ )" we need the Grothendieck inequality for JB*-triples (see [17, Theorem 10]). Proposition 2.2 in [28] is also a direct consequence of the previous Theorem.

We have already given many examples of Banach spaces $X$ for which the topologies $\rho(X)$ and $s^{*}\left(X, X^{*}\right)$ coincide on bounded subsets of $X$. This class of examples includes $\mathrm{JB}^{*}$-triples, $\mathrm{C}^{*}$-algebras, and $\mathrm{JB}^{*}$-algebras. We shall now show that this behaviour remains true for some other classes of Banach spaces. We shall also study the relationships between quasi completely continuous operators and some other classes of operators which have been investigated by many different authors.

We shall start with reflexive Banach spaces. The next remark shows that, for a reflexive Banach space $X$ the topologies $\rho(X)$ and $s^{*}\left(X, X^{*}\right)$ coincide on bounded subsets of $X$ if, and only if, $X$ is isomorphic to a Hilbert space.

Remark 5.11. Let $R$ be a reflexive Banach space. Suppose that the topologies $m\left(R, R^{*}\right)$ and $s^{*}\left(R, R^{*}\right)$ coincide on bounded subsets of $R$. In this case, Proposition 5.2,b), applied to the operator $T: R \rightarrow R, x \mapsto 2 x$, shows that there exists a bounded linear operator $G$ from $R$ into a Hilbert space $H$ satisfying

$$
2\|x\| \leq\|G(x)\|+\|x\|
$$

for all $x \in R$, which implies that $R$ is isomorphic to a Hilbert space. That is, for a reflexive Banach space $R$, the topologies $m\left(R, R^{*}\right)$ and $s^{*}\left(R, R^{*}\right)$ coincide on bounded subsets of $R$ if and only if $R$ is isomorphic to a Hilbert space. On the other hand, it is clear that the $m\left(R, R^{*}\right)$ topology coincides with the norm topology on every reflexive Banach space $R$. 
We shall now deal with $\ell_{p}$ spaces.

Remark 5.12. - Let $2<p<+\infty$. Pitt's Theorem tells us that every operator $T: \ell_{p} \longrightarrow \ell_{2}$ is compact and hence completely continuous (compare [14, Proposition 2.c.3]). Therefore, every weakly-null sequence, $\left(x_{n}\right) \subset \ell_{p}$, is $s^{*}\left(\ell_{p}, \ell_{p}^{*}\right)$-null. Since $s^{*}\left(X, X^{*}\right)$-null sequences are weakly-null for every Banach space $X$, we get that on $\ell_{p}(p>2)$ weakly-null and $s^{*}\left(\ell_{p}, \ell_{p}^{*}\right)$-null sequences coincide. It follows that, for every Banach space $X$ and every $p>2$, an operator

$$
T: \ell_{p} \longrightarrow X
$$

is completely continuous if and only if it is quasi completely continuous.

- Note that the identity mapping on $\ell_{p}$ is a weakly compact operator, hence unconditionally converging, by the Orlicz-Pettis Theorem. However, it is not completely continuous (the canonical basis $\left(e_{n}\right)$ is weakly-null but not normnull), and therefore it is not quasi completely continuous. This provides a simple example of an unconditionally converging but not quasi completely continuous operator.

- We have already seen in the previous remark that the topologies $s^{*}\left(\ell_{p}, \ell_{p}^{*}\right)$ and $m\left(\ell_{p}, \ell_{p}^{*}\right)$ do not coincide on bounded subsets of $\ell_{p}(1 \leq p<\infty, p \neq 2)$.

- The above paragraphs show that the hypothesis of coincidence between the $s^{*}(X)$ topology and the right topology on bounded subsets can not be omitted in Theorem $5.7, b) \Rightarrow c$ ), even when $X$ has property $(V)$ (observe that every reflexive Banach space has property $(V)$ ).

\section{REFERENCES}

[1] Akemann, C. A., The dual space of an operator algebra, Trans. Amer. Math. Soc. 126 286-302 (1967).

[2] Akemann, C. A., Dodds, P. G., Gamlen, J. L. B., Weak compactness in the dual space of $C^{*}$-algebra, J. Funct. Anal. 10, 446-450 (1972).

[3] Bartle, R. G., Dunford, N., and Schwartz, J., Weak compactness and vector measures, Canad. J. Math. 7, 289-305 (1955).

[4] Barton, T. and Friedman Y., Grothendieck's inequality for JB*-triples and applications, J. London Math. Soc. (2) 36, 513-523 (1987).

[5] Bourgain, J. and Pisier, G., A construction of $\mathcal{L}_{\infty}$-spaces and related Banach spaces. Bol. Soc. Brasil. Mat. 14 109-123 (1983).

[6] Chu, Ch.-H. and Mellon, P., JB*-triples have Pełczyński's Property V, Manuscripta Math. 93, no. 3, 337-347 (1997).

[7] Davis, W. J., Figiel, T., Johnson W. B., and Pełczyński, A., Factoring weakly compact operators, J. Funct. Anal. 17, 311-327 (1974).

[8] Diestel, J., Sequences and series in Banach spaces, Graduate Texts in Mathematics, 92. Springer-Verlag, New York, 1984.

[9] Dineen, S., The second dual of a JB*-triple system, In: Complex analysis, functional analysis and approximation theory (ed. by J. Múgica), 67-69, (North-Holland Math. Stud. 125), North-Holland, Amsterdam-New York, 1986.

[10] Grothendieck, A.: Résumé de la théorie métrique des produits tensoriels topologiques, Bol. Soc. Mat. Sao Paolo 8, 1-79 (1956).

[11] Haagerup, U., The Grothendieck inequality for bilinear forms on $\mathrm{C}^{*}$-algebras, $A d v$. Math. 56, 93-116 (1985). 
[12] Jarchow, H., Locally Convex Spaces, Teubner, Stuttgart, 1981.

[13] Jarchow, H., On weakly compact operators on $C^{*}$-algebras, Math. Ann. 273, 341-343 (1986).

[14] Lindenstrauss, J. and Tzafriri, L., Classical Banach spaces. I. Sequence spaces, Ergebnisse der Mathematik und ihrer Grenzgebiete, Vol. 92. Springer-Verlag, Berlin-New York, 1977.

[15] Peralta, A. M., Some remarks on weak compactness in the dual space of a JB*-triple, Thoku Math. J. 58, 149-159 (2006).

[16] Peralta, A. M., Topological characterisation of weakly compact operators revisited, Extracta Math., 22 (2), 215-223 (2007).

[17] Peralta, A. M. and Rodríguez Palacios, A., Grothendieck's inequalities for real and complex JBW*-triples, Proc. London Math. Soc. (3) 83, no. 3, 605-625 (2001).

[18] Peralta, A. M., Villanueva, I., Wright, J. D. M. and Ylinen, K., Topological characterisation of weakly compact operators, J. Math. Anal. Appl. 325, no. 2, 968-974 (2007).

[19] Pisier, G., Grothendieck's theorem for non commutative $\mathrm{C}^{*}$-algebras with an appendix on Grothendieck's constant, J. Funct. Anal. 29, 397-415 (1978).

[20] Rodríguez Palacios A., On the strong* topology of a JBW*-triple, Quart. J. Math. Oxford (2) 42, 99-103 (1989).

[21] Ruess, W. [Weakly] Compact Operators and DF Spaces, Pacific J. Math 98 (2) (1982) 419-441.

[22] Saitô, K., On the preduals of $W^{*}$-algebras, Tôhoku Math. J. (2) 19, 324-331 (1967).

[23] Sakai, S., $C^{*}$-algebras and $W^{*}$-algebras, Springer-Verlag, Berlin 1971.

[24] Takesaki, M., On the conjugate space of operator algebra. Tôhoku Math. J. (2) 10, 194-203 (1958).

[25] Wong, N. Ch. and Wong, Y.-Ch., Topologies and bornologies determined by operator ideals, Math. Ann. 282, no. 4, 587-614 (1988).

[26] Wright, J. D. M., Linear representations of bilinear forms on operator algebras, Expo. Math., volume 16, 75-84 (1998).

[27] Wright, J. D. M., Right topology for Banach spaces and weak compactness, Atti. Semin. Mat. Fis. Univ. Modena. Reggio Emilia vol 55, 1-11 (2007).

[28] Wright, J. D. M. and Ylinen, K., Multilinear maps on products of operator algebras, J. Math. Anal. Appl. 292 no. 2, 558-570 (2004).

E-mail address: aperalta@ugr.es

Departamento de Análisis Matemático, Facultad de Ciencias, Universidad de Granada, 18071 Granada, Spain.

E-mail address: ignaciov@mat.ucm.es

Departamento de Análisis Matemático, Facultad de Matemáticas, UniverSidAd Complutense de Madrid, MAdrid 28040, Spain

E-mail address: j.d.m.wright@abdn.ac.uk

Mathematical Sciences, University of Aberdeen, Aberdeen AB 24 3Ue, ScotLAND

E-mail address: ylinen@utu.fi

Department of Mathematics, University of Turku, Fin-20014 Turku, FinLAND 